\title{
A Polymerase-Activating Host Factor, YajQ, Bound to the Bacteriophage $\phi 6$ Capsid
}

\author{
J. Bernard Heymann ${ }^{1}$, Daniel Nemecek ${ }^{2}$, Rick Huang ${ }^{3}$, Naiqian Cheng ${ }^{1}$, Jian Qiao ${ }^{4}$, Leonard Mindich \\ ${ }^{4}$ and Alasdair C. Steven ${ }^{1}$ \\ ${ }^{1}$ Laboratory of Structural Biology Research, NIAMS, NIH, 50 South Dr, Bethesda, MD 20892. \\ ${ }^{2}$ Electron Microscopy and Imaging Unit, Sanofi-Pasteur, 69280 Marcy L’Etoile, Lyon, France. \\ ${ }^{3}$ Cryo-EM Facility, Janelia Research Campus, HHMI Ashburn, Virginia 20147. \\ ${ }^{4}$ Department of Microbiology, PHRIC, University of Medicine and Dentistry of New Jersey, 225 \\ Warren Street, Newark, NJ 07103.
}

Bacteriophage $\phi 6$ has a tripartite double-stranded RNA genome packaged inside three concentric shells. The three segments code for the various viral proteins, with the large (L) segment containing the genes for producing new procapsids. These are the P1 protein making up the inner shell or capsid, the RNAdependent RNA polymerase, P2, the packaging motor, P4, and the packaging facilitator, P7. The transcription of the $\mathrm{L}$ segment is regulated such that it is active at an early stage when the virus infects its host, Pseudomonas syringae, while it is suppressed late in infection. This regulation is associated with a difference in the 5 ' terminus of the L segment as compared to the medium (M) and small (S) segments (the 5' terminus starts with GU in $\mathrm{L}$ and $\mathrm{GG}$ in $\mathrm{M}$ and $\mathrm{S}$ ). It was discovered that a host protein, YajQ, is required for the early stage activation of L segment transcription [1]. YajQ binds to the outside of the capsid, while the transcription activity occurs inside the capsid. We are interested in how this communication across the capsid shell occurs.

The RNA-packaged mature capsids were prepared from the Pseudomonas syringae strain LM4383 [1]. The capsids were plunge-frozen and imaged in a Polara FEG microscope (FEI) operated at $300 \mathrm{kV}$ and recorded on a K2 Summit direct electron detector (Gatan, Inc.) at a pixel size of $1.03 \AA$ as follows: 10 frames were collected in counting mode at $0.3 \mathrm{~s} /$ frame at a dose rate of $\sim 10 \mathrm{e}^{-} /\left(\mathrm{s} \AA^{2}\right)$ for a total dose of $\sim 30 \mathrm{e}^{-} / \AA^{2}$, at $1.0-2.0 \mu \mathrm{m}$ underfocus. The frames were aligned (Figure 1A) and reconstructions generated with icosahedral symmetry from 5272 particle images to $6.2 \AA$ (Figure 1B,C) using Bsoft [2].

The reconstruction shows densities bound to the outer surface of the capsid shell (arrows in Figure 1B,C). A model was generated from the crystal structure of the highly homologous YajQ (51\% identity) from Haemophilus influenzae [3]. The model was adjusted by limited flexible fitting into the densities, with the $\mathrm{N}$ - and $\mathrm{C}$-terminal ends poorly represented (Figure 2, green star). The structures of the two subunits, $\mathrm{P} 1_{\mathrm{A}}$ and $\mathrm{P} 1_{\mathrm{B}}$ [4], were also fitted into the capsid shell, revealing an extra density associated with subunit $\mathrm{P} 1_{\mathrm{A}}$ (Figure2, blue star). Other than this, no sizeable conformational change is apparent in the capsid shell, indicating that the binding of YajQ induces a more subtle change to influence the polymerase on the inside of the capsid. The location of the polymerase inside the packaged capsid remains unknown. We aim to unravel how YajQ activates the polymerase in future studies. 


\section{References:}

[1] X Qiao, et al., Proc Natl Acad Sci U S A 105 (2008), p. 15956-60.

[2] JB Heymann and DM Belnap, J Struct Biol 157 (2007), p. 3-18.

[3] A Teplyakov, et al., J Struct Funct Genomics 4 (2003), p. 1-9.

[4] D Nemecek, et al., Structure 21 (2013), p. 1374-83.
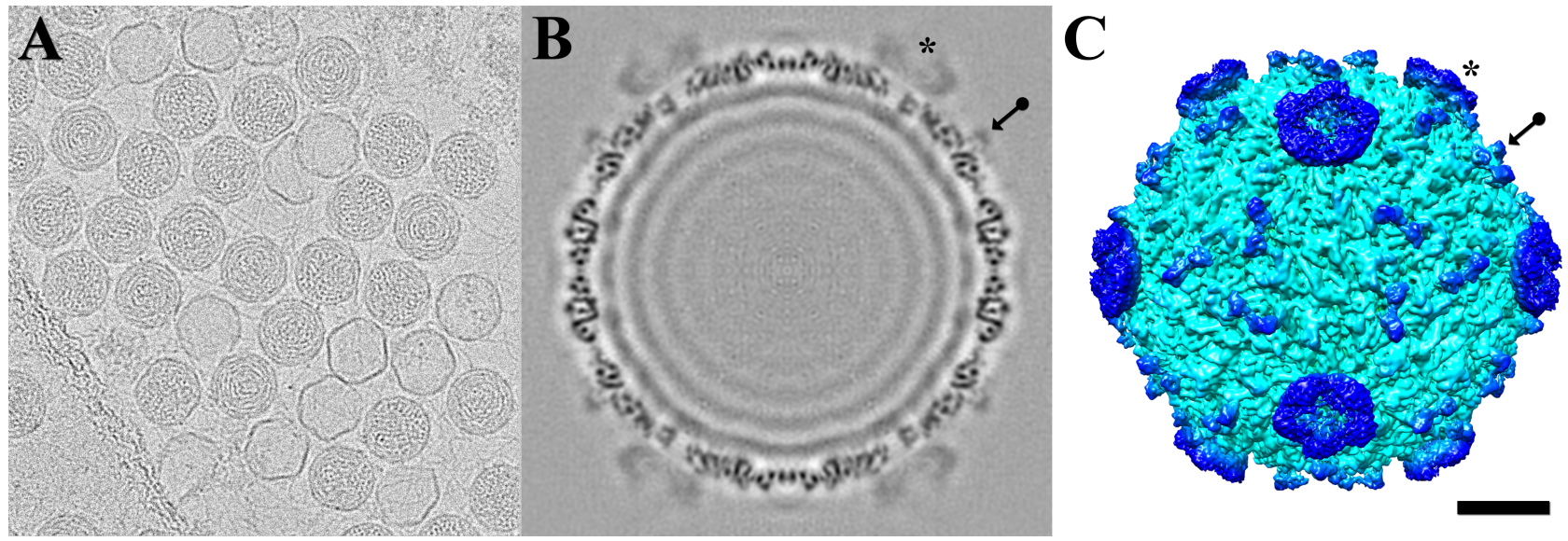

Figure 1. (A) Cryo-electron micrograph of a preparation of packaged $\phi 6$ capsids decorated with YajQ. Some loss of RNA resulted in empty capsids. Central slice (B) and isosurface (C) of a reconstruction at $6.2 \AA\left(\mathrm{FSC}_{0.3}\right)$, indicating YajQ (arrow) and the packaging motor, P4 (star). Scale bar: $100 \AA$.

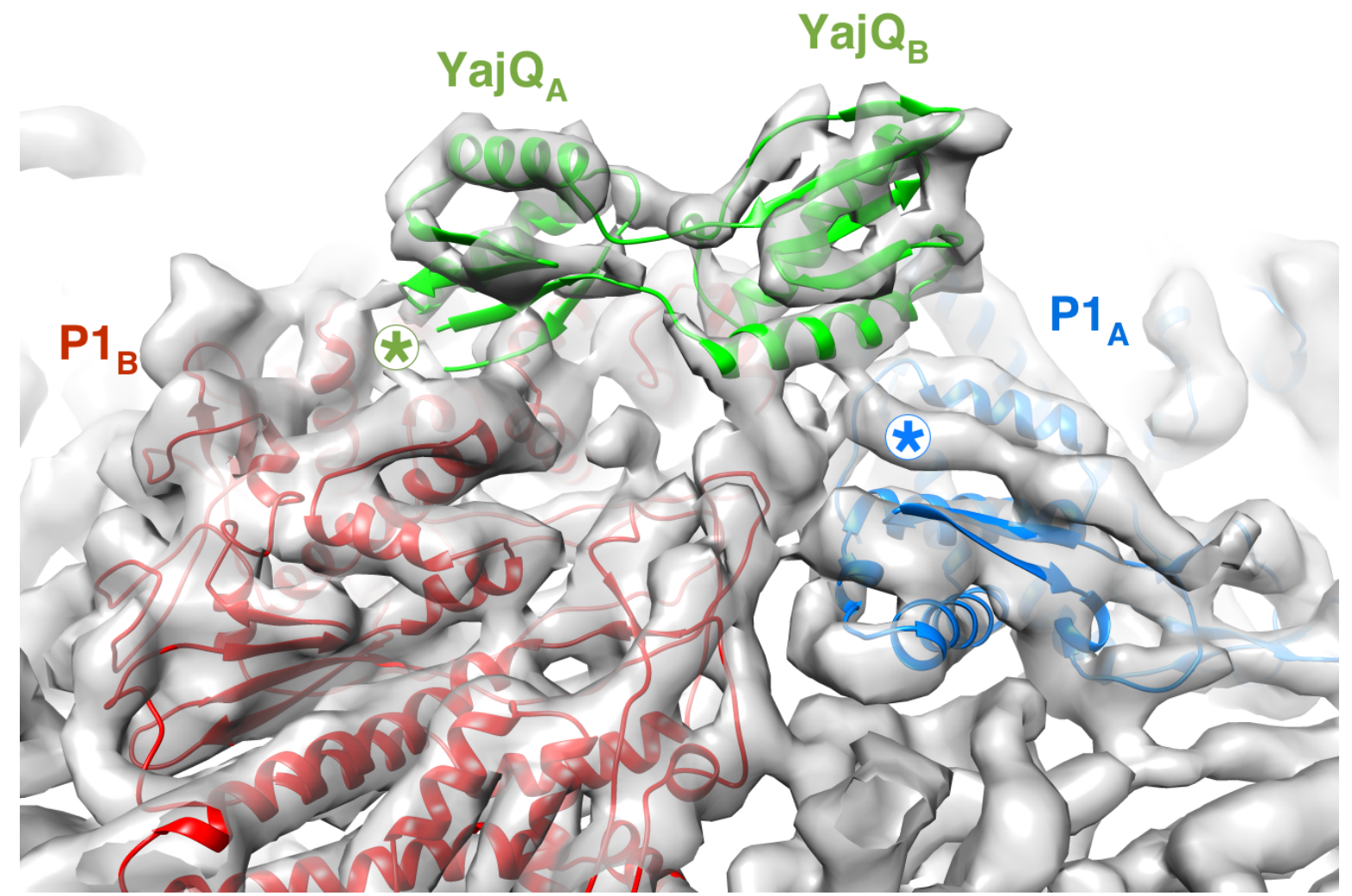

Figure 2. Fit of a homology model of YajQ (green) into the density attached to the capsid. Also shown are the two capsid subunits, $\mathrm{P} 1_{\mathrm{A}}$ (blue) and $\mathrm{P} 1_{\mathrm{B}}(\mathrm{red})$. The $\mathrm{N}$ - and C-termini of YajQ do not fit into density (green star), suggesting an alternative organization not clear in this map. Curiously, the blue star indicates additional density associated with the $\mathrm{P} 1_{\mathrm{A}}$ subunit not seen in reconstructions without YajQ. 